\title{
Transcription is required for establishment of germline methylation marks at imprinted genes
}

\author{
Mita Chotalia, ${ }^{1,4}$ Sebastien A. Smallwood, ${ }^{1}$ Nico Ruf, ${ }^{1}$ Claire Dawson, ${ }^{1}$ Diana Lucifero, ${ }^{1}$ \\ Marga Frontera, ${ }^{1}$ Katherine James, ${ }^{2}$ Wendy Dean, ${ }^{1}$ and Gavin Kelsey ${ }^{1,3,5}$ \\ ${ }^{1}$ Laboratory of Developmental Genetics and Imprinting, The Babraham Institute, Cambridge CB22 3AT, United Kingdom; \\ ${ }^{2}$ Emmanuel College, University of Cambridge, Cambridge CB2 3AP, United Kingdom; ${ }^{3}$ Centre for Trophoblast Research, \\ University of Cambridge, Cambridge, United Kingdom
}

\begin{abstract}
Genomic imprinting requires the differential marking by DNA methylation of genes in male and female gametes. In the female germline, acquisition of methylation imprint marks depends upon the de novo methyltransferase Dnmt3a and its cofactor Dnmt3L, but the reasons why specific sequences are targets for Dnmt3a and Dnmt3L are still poorly understood. Here, we investigate the role of transcription in establishing maternal germline methylation marks. We show that at the Gnas locus, truncating transcripts from the furthest upstream Nesp promoter disrupts oocyte-derived methylation of the differentially methylated regions (DMRs). Transcription through DMRs in oocytes is not restricted to this locus but occurs across the prospective DMRs at many other maternally marked imprinted domains, suggesting a common requirement for transcription events. The transcripts implicated here in gametic methylation are protein-coding, in contrast to the noncoding antisense transcripts involved in the monoallelic silencing of imprinted genes in somatic tissues, although they often initiate from alternative promoters in oocytes. We propose that transcription is a third essential component of the de novo methylation system, which includes optimal CpG spacing and histone modifications, and may be required to create or maintain open chromatin domains to allow the methylation complex access to its preferred targets.
\end{abstract}

[Keywords: Genomic imprinting; DNA methylation; oocytes; transcription]

Supplemental material is available at http://www.genesdev.org.

Received July 4, 2008; revised version accepted November 12, 2008.

Genomic imprinting in mammals is controlled by discrete elements, termed imprinting control regions (ICRs), that become methylated in male or female gametes and retain this modification as a memory of parental origin in the somatic tissues of the conceptus, where it is acted upon to cause monoallelic silencing of imprinted genes (Reik and Walter 2001a). All imprinted domains possess differentially methylated regions (DMRs) with germlinederived methylation (although only a subset have been functionally verified as ICRs), and most such marks are laid down in the female germline (Reik and Walter 2001b). Establishment of methylation imprints in oocytes occurs postnatally, in growing oocytes arrested in meiosis I (Lucifero et al. 2004; Hiura et al. 2006), and requires the cooperative activities of the DNA methyltransferase Dnmt3a and its nonenzymatic cofactor Dnmt3L

\footnotetext{
${ }^{4}$ Present address: Genome Function Group, MRC Clinical Sciences Centre, Imperial College School of Medicine, Hammersmith Hospital Campus, Du Cane Road, London W12 0NN, United Kingdom.

${ }^{5}$ Corresponding author.

E-MAIL gavin.kelsey@bbsrc.ac.uk; FAX 44-1223-496022.

Article is online at http://www.genesdev.org/cgi/doi/10.1101/gad.495809.
}

(Bourc'his et al. 2001; Hata et al. 2002; Kaneda et al. 2004). Dnmt3L acts in the maternal germline apparently specifically for the establishment of DMRs (Bourc'his et al. 2001; Hata et al. 2002).

DMRs are CG-rich sequences that fulfill the criteria of CpG islands (CGIs), but the properties that distinguish them from the majority of CGIs and result in their gametic methylation remain to be fully elucidated. At a sequence level, DMRs frequently contain tandem repeats (Neumann et al. 1995; Hutter et al. 2006; Reinhart et al. 2006), whose functional significance is supported by some transgenic studies (Neumann et al. 1995; Reinhart et al. 2006); however, not all maternal germline DMRs possess this feature (Arnaud et al. 2003), and tandem repeats also occur in the CGIs of apparently nonimprinted loci (Yamada et al. 2004). Structural studies have revealed that the Dnmt3a/3L complex methylates DNA templates in a periodic pattern, with an optimal spacing of CpGs of 8-10 base pairs (bp) (Jia et al. 2007; Jurkowska et al. 2008). Furthermore, it has been suggested that this periodicity characterizes maternal germline DMRs (Jia et al. 2007). This finding could help explain the prevalence 
of tandem repeats in DMRs, but the degree to which an 8- to 10-bp CpG periodicity is specific to DMRs remains to be confirmed (Ferguson-Smith and Greally 2007). Biochemical studies have shown that Dnmt3L interacts with histone $\mathrm{H} 3$, but only when $\mathrm{H} 3$ is unmethylated at Lys 4 (Ooi et al. 2007), revealing that the chromatin signature of target sequences is also an important factor. Methylated H3K4 is recognized as a mark of active promoters and transcription units (Bernstein et al. 2005; Kim et al. 2005).

Other evidence indicates that sequences outside of DMRs can make an essential contribution to methylation. DMRs on transgenes often fail to adopt imprinted methylation, unless in the context of large constructs or in the presence of additional sequence elements (e.g., Sleutels and Barlow 2001). In two human imprinted gene syndromes, deletions many kilobases from DMRs have been shown to disrupt methylation. Maternally transmitted microdeletions upstream of the SNRPN DMR cause Angelman syndrome (AS) as a result of loss of methylation (LoM) of this DMR; the overlap between deletions defines a $<1-k b$ region $35 \mathrm{~kb}$ upstream of the DMR that appears to be required for methylation (Buiting et al. 1999). In Pseudohypoparathyroidism type $1 \mathrm{~b}$ (PHP1b), an endocrine disorder associated with imprinting mutations in the GNAS locus, maternal allele methylation of the GNAS DMRs is disrupted by deletions in the neighboring NESP55 or STX16 genes, the latter being $\sim 220 \mathrm{~kb}$ from the affected DMR (Bastepe et al. 2003, 2005). How such remote elements are involved in methylation of the associated DMRs is unclear, but their existence strongly suggests that DMR sequence alone is insufficient.

A potential factor that has not been explored in any detail is transcription, although there are lines of evidence suggestive of a role for transcription in methylation of germline DMRs, and a transcription-based mechanism could provide a unifying explanation for the observations above. Many maternal germline DMRs are contained within transcription units, including those at four recently evolved imprinted retrotransposons (Wood et al. 2007), and in general, intragenic CGIs are more likely to be methylated than those at promoters (Yamada et al. 2004; Illingworth et al. 2008). In a transgene study, the Air DMR was appropriately methylated in some mouse lines when located in an intron of an Aprt transgene (Sleutels and Barlow 2001). Finally, in somatic cells, noncoding antisense transcripts have been implicated in methylation of the "somatic" DMRs through which they transcribe (Sleutels et al. 2002; Williamson et al. 2006; Shin et al. 2008), although the mechanistic aspects of this remain to be established (Pauler et al. 2007). Here, we directly test the hypothesis that transcription is required for establishment of methylation of DMRs in female gametes. At several maternally marked imprinted domains, we detect transcripts traversing the DMRs in growing oocytes prior to and at the time of de novo methylation. Using the mouse Gnas locus as a model, we show that disruption of the Nesp transcript, which initiates furthest upstream in this imprinted domain, prevents normal establishment of methylation of the germline DMRs. In striking contrast to monoallelic silencing of imprinted genes in somatic tissues by antisense transcription, the transcripts we believe to be involved in gametic methylation are protein-coding. Our results thus reveal a novel, transcriptionally mediated component in imprint establishment. Transcription across DMRs in germ cells may be essential for remodeling histone modifications or to create chromatin domains permissive for de novo methylation. These findings provide a basis for understanding how new imprinted loci evolve and a novel molecular explanation for imprinting errors.

\section{Results}

Transcription across the Gnas locus DMRs in growing oocytes

We are using the mouse Gnas locus to investigate mechanisms of imprint establishment. The locus comprises a series of overlapping, imprinted, protein-coding transcripts Gnas, Gnasxl, and Nesp and the imprinted noncoding transcripts Nespas and $1 \mathrm{~A}$ (Fig. 1A). An extensive DMR covering the Gnasxl and Nespas promoters and a second DMR covering the $1 \mathrm{~A}$ promoter govern monoallelic expression of these transcripts (Williamson et al. 2004, 2006; Liu et al. 2005). Both DMRs are methylated on the maternal allele and acquire methylation in oocytes by a Dnmt3L dependent mechanism (Liu et al. 2005; Arnaud et al. 2006). An additional DMR with paternal allele methylation covers the Nesp exons but is methylated after fertilization (i.e., a somatic DMR), while the Gnas promoter is located within a constitutively unmethylated CGI (see Fig. 1B; Liu et al. 2000). This pattern of methylation makes Gnas a particularly informative imprinted cluster in which to investigate how imprints are established: Any model must explain why adjacent elements like the $1 A$ DMR and Gnas CGI are marked differently. We are especially interested in the Nesp transcript (which in somatic tissues is expressed only from the maternal allele), because its start site is furthest upstream in the locus, such that transcription events initiating at the Nesp promoter traverse the whole cluster, including the two germline DMRs. In addition, maternally transmitted deletions of the NESP region in humans are associated with LoM of the NESPAS/GNASXL and $1 A$ DMRs in some PHP1b families (Bastepe et al. 2005). To establish a possible role for Nesp transcription in methylation acquisition, we first showed by RT-PCR, using primer sets that detect transcription events that traverse the maternal germline DMRs at Nespas/Gnasxl and 1A, that Nesp transcripts could be detected in growing oocytes obtained at 5, 10, and 15 d post-partum (dpp) (Fig. 1C). These germline DMRs start to become methylated from day 15 (Fig. 1B). In contrast, Nespas, Gnasxl, and $1 A$ transcripts were not detected by RT-PCR during oocyte growth under the same conditions in which Nesp was readily detected (Fig. 1C), suggesting that the Nespas, Gnasxl, and $1 A$ promoters are inactive even before the onset of methylation of their DMRs, whereas Gnas transcripts were present throughout. Interestingly, by 5'RACE analysis, we found that the start site utilized by Nesp transcripts in oocytes was distinct 
A
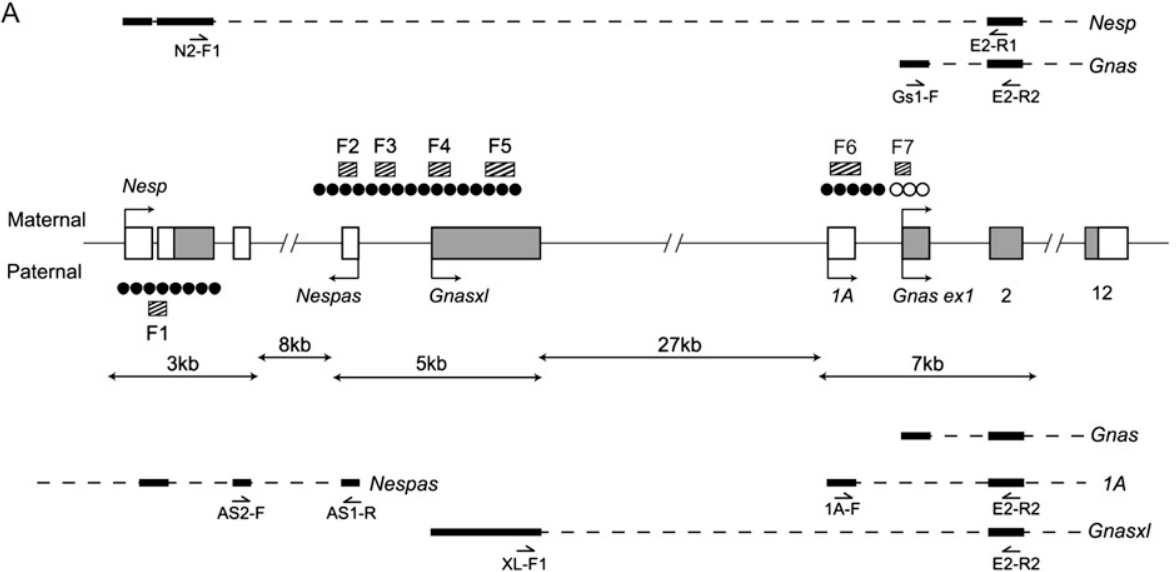

B
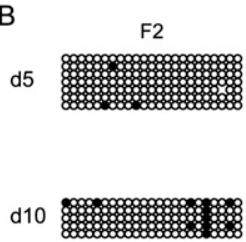

d15

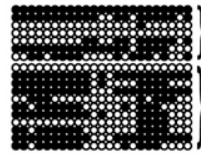

MII

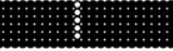

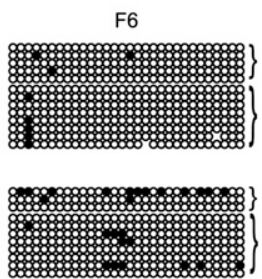
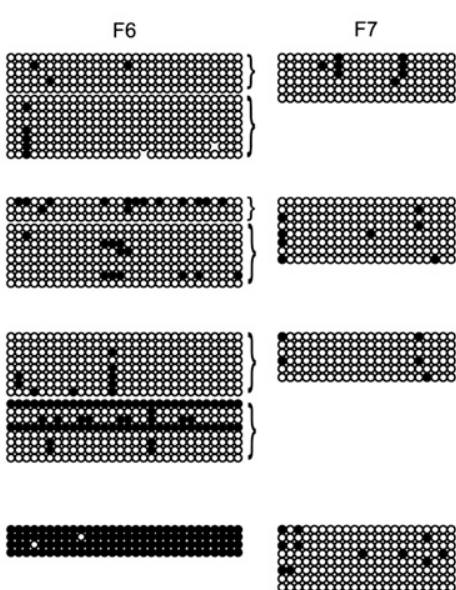

C
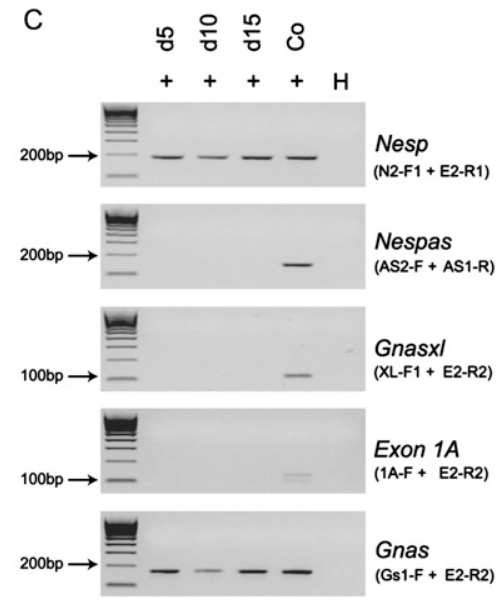

Figure 1. The Nesp transcript is detected in growing oocytes coincident with establishment of methylation of germline DMRs in the Gnas locus. (A) Scheme of the mouse Gnas locus (Plagge and Kelsey 2006), showing the organization of the overlapping, protein-coding transcripts Nesp, Gnasxl, and Gnas and the noncoding Nespas and $1 A$ transcripts. Transcripts expressed from the maternal allele are indicated above the line, from the paternal allele below the line; Gnas exhibits tissue-specific imprinting, with repression of the paternal allele in a subset of tissues. The location of the DMRs is shown by the rows of filled circles on the methylated allele; the Gnas promoter resides in a biallelically unmethylated CGI (open circles). The positions of the PCR products for bisulphite analysis in $B$ and Figures 3 and 4 are indicated by the hatched bars, and the primers for the RT-PCR assays in $C$ and Figure 2 by labeled arrows. $(B)$ Bisulphite sequences of the Nespas/Gnasxl and 1A DMRs in oocytes isolated at postnatal days 5, 10, and 15 and mature metaphase II (MII) oocytes from adult females. Each row represents the $\mathrm{CpG}$ sites of an individual sequenced clone, with filled circles depicting methylated CpG sites (missing circles represent CpGs for which sequence was ambiguous). Sequences obtained from two independent bisulphite treatments are indicated by bracketed sets of methylation profiles. Methylation of these DMRs in MII oocytes has been described previously (Liu et al. 2000; Coombes et al. 2003). (C) RT-PCRs for Nesp, Nespas, Gnasxl, 1A, and Gnas transcripts in day 5, 10, 15 oocytes. Co indicates amplification control with cDNA from an E13.5 embryo; $\mathrm{H}$ indicates no template control. The left lanes on each gel show a 100 -bp marker ladder, with the 100- or 200-bp markers indicated.

from the major start site used in somatic tissues (Supplemental Fig. 1). In conclusion, transcription from Nesp occurs across the DMRs in oocytes before and possibly during methylation establishment, while the DMRs themselves may correspond to inactive promoters.

\section{A targeted allele to truncate Nesp transcription}

As a functional test of the role of Nesp transcription, we designed a targeted allele to prematurely terminate transcription. We replaced the splice donor site of the second Nesp exon with a cassette comprising the final exon, polyadenylation, and downstream sequences of the rabbit $\beta$-globin gene (Fig. 2A,B); this cassette has been used effectively elsewhere to terminate noncoding RNAs to examine their effect in somatic tissues on imprinted monoallelic expression (Sleutels et al. 2002; Shin et al. 2008). After germline transmission of the targeted allele and excision of the selection markers (this allele is termed Nesp ${ }^{\text {trun }}$ ), we checked for the effect on the Nesp transcript. RT-PCR assays of newborn brain tissue (where Nesp is highly expressed) failed to detect the normal Nesp transcript after maternal transmission of the Nesp ${ }^{\text {trun }}$ allele (Fig. 2C). Complete ablation of the normal Nesp transcript was verified by real-time PCR (data not shown), and 3'RACE analysis confirmed that the expected premature polyadenylation event had 
Chotalia et al.

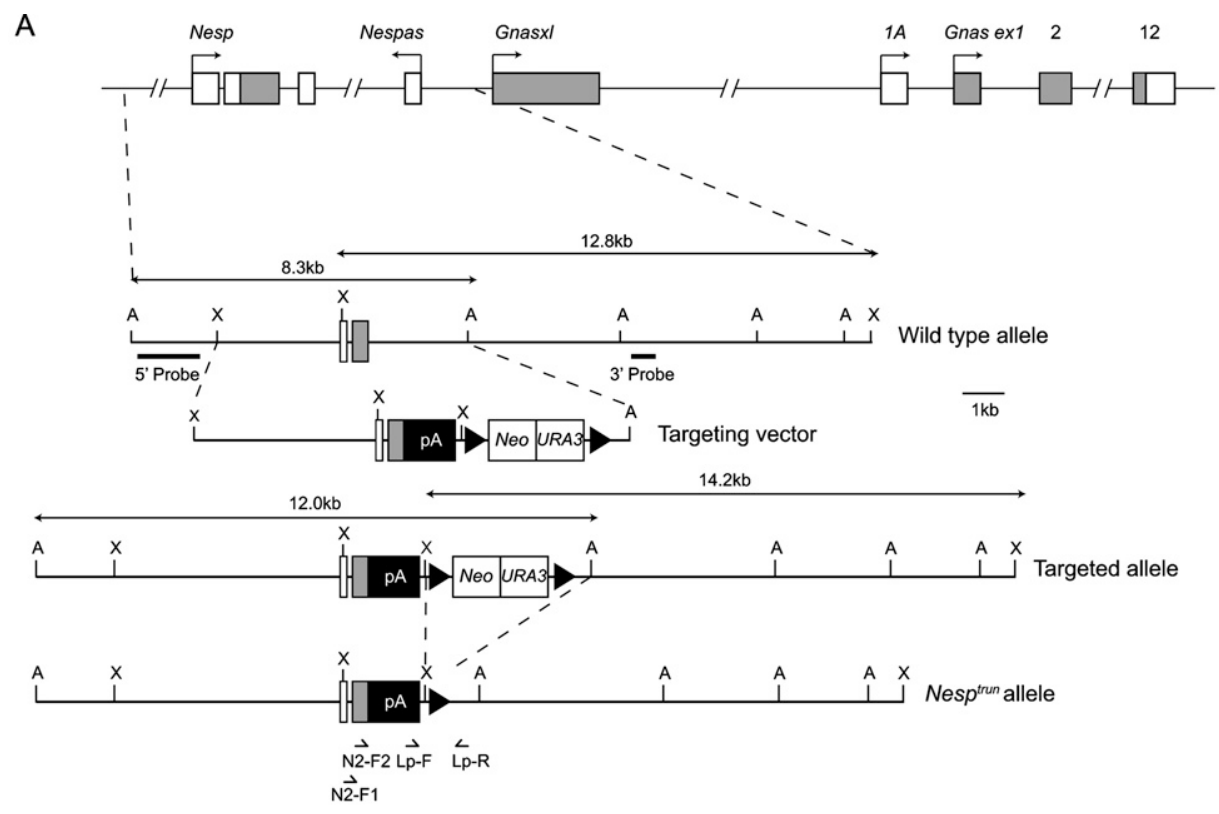

B
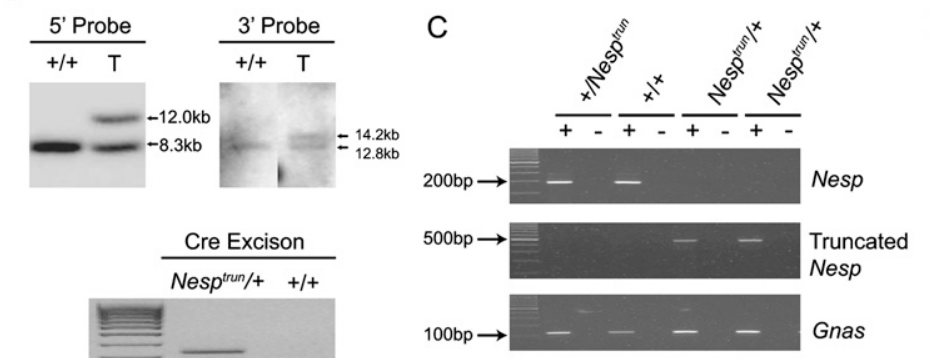

D
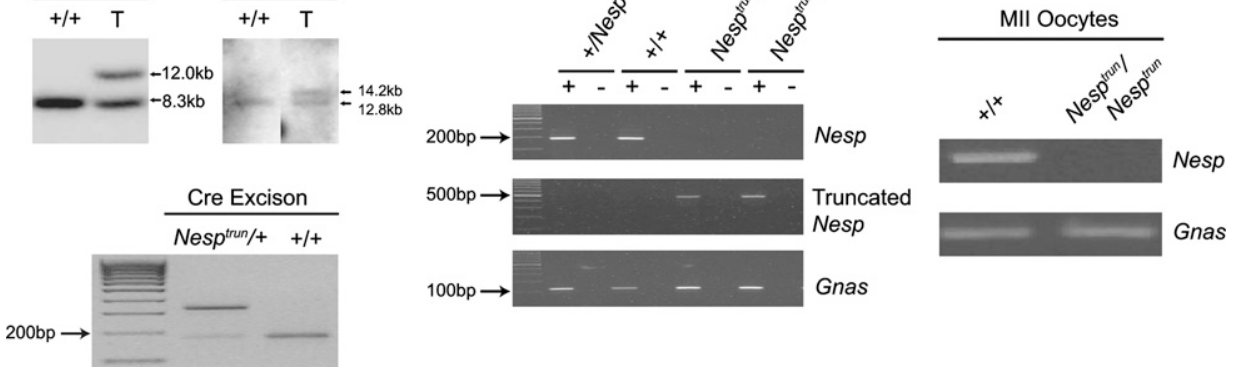

Figure 2. Truncation of Nesp transcription by insertion of a termination cassette. $(A)$ Scheme of the targeting vector in relation to the Gnas locus, the targeted allele, and the Nesp ${ }^{\text {trun }}$ allele after Cre-mediated excision of the selectable marker cassette. The rabbit $\beta$-globin cassette is represented by the black box, NeO ${ }^{R}$ and URA3 selection cassettes as open boxes, and loxP sites as black arrowheads. The location of restriction sites AflII (A) and XhoI (X) for Southern blot detection of targeting events is given. (B) Southern blots of wild-type $(+/+)$ and targeted (T) embryonic stem (ES) cell clones detecting correct recombination events by hybridization with $5^{\prime}$ and $3^{\prime}$ probes in AflII and XhoI digests, respectively. Below, PCRs using primers Lp-F and Lp-R to verify the Cre-mediated excision event after germline transmission (confirmed by sequencing of the PCR product) (data not shown) and N2-F2 and Lp-R to detect the wild-type allele. (C) RTPCR analysis of neonatal brain RNA after paternal $\left(+/ N e s p^{\text {trun }}\right)$ and maternal $\left(N e s p^{\text {trun }} /+\right)$ transmission of the Nesp ${ }^{\text {trun }}$ allele. RT-PCRs for Nesp and Gnas were done primers using N2-F1 + E2-R1 and Gs1-F + E2-R2, respectively, as in Figure 1A. The RT-PCR for Nesp therefore assays the presence of wild-type, full-length Nesp transcripts, which are absent after maternal transmission. The panel labeled Truncated Nesp is a 3'RACE assay (primer N2-F1 and 3'RACE primer GR-R2) for the presence of prematurely terminated Nesp transcripts; sequencing these PCR products verified that the desired truncation event had occurred (Supplemental Fig. 2). (D) RT-PCR assay (primers as above) showing the absence of full-length Nesp transcripts in MII oocytes from a Nesp ${ }^{\text {trun }}$ homozygous female.

occurred (Fig. 2C). Finally, analysis of oocytes from Nesp $p^{\text {trun/trun }}$ homozygous females revealed that the Nesp transcript that normally traverses the DMRs was undetectable (Fig. 2D) and the expected truncation product was detected by 3'RACE (Supplemental Fig. 2), suggesting that transcription had indeed been terminated upstream of the germline DMRs.

\section{Nesp truncation causes loss of germline methylation at} the Gnas locus

To examine whether truncation of Nesp altered the methylation of the germline DMRs, we first analyzed
DNA of tissues from newborn pups (denoted Nesp $\mathrm{trun} /+^{+}$) inheriting the Nesp ${ }^{\text {trun }}$ allele maternally. Nesp ${ }^{\text {trun }}$ carrier females were crossed with C57BL/6J males or with males carrying the Gnas region of chromosome 2 from Mus spretus to enable discrimination of parental alleles using sequence polymorphisms. In the carrier females, the Nesp ${ }^{\text {trun }}$ allele was of paternal origin, so that their progeny represented the first maternal transmission of the truncation allele. All samples were tested by combined bisulphite and restriction analysis (COBRA) (representative COBRA results are shown in Supplemental Fig. $3)$, and PCR products from a subset were cloned and 
sequenced to determine complete methylation profiles. As shown in Figures $3 \mathrm{~A}$ and $4 \mathrm{~A}$, pups inheriting the Nesp ${ }^{\text {trun }}$ allele from females showed LoM of the maternal germline DMRs at Nespas/Gnasxl and/or 1A. Where informative polymorphisms were present (as in amplicons F3 and F5 in Fig. 3A), it was possible to confirm in the bisulphite sequence profiles that the normally methylated maternal allele had indeed lost methylation. Unexpectedly, the Nesp $p^{\text {trun/+ }}$ mutants differed in their degree of LoM (Fig. 3A,B), and this was the case even among littermates. All pups inheriting the Nesp ${ }^{\text {trun }}$ allele maternally fully lacked methylation of the $1 A$ DMR (amplicon F6 in Fig. 3). In $68 \%$ of Nesp $p^{\text {trun/+ }}$ pups, there was also
LoM of the Gnasxl exon and promoter (amplicons F4, F5), and in $24 \%$, LoM extended further upstream to the Nespas first exon (amplicon F2). This last category therefore had complete LoM of all the maternal germline DMRs (e.g., mutants \#A25 and \#A62 in Fig. 3A). In this class of Nesp ${ }^{\text {trun/+ }}$ mutant, we would expect expression of the antisense Nespas on the maternal allele to be derepressed, which should result in acquisition of methylation on the maternal allele of the somatic DMR at Nesp (Williamson et al. 2006), and this was found to be the case (amplicon F1 in Fig. 3A). No LoM at any DMR was observed in seven wild-type littermate controls; paternal transmission of the Nesp truncation also had no effect on

A
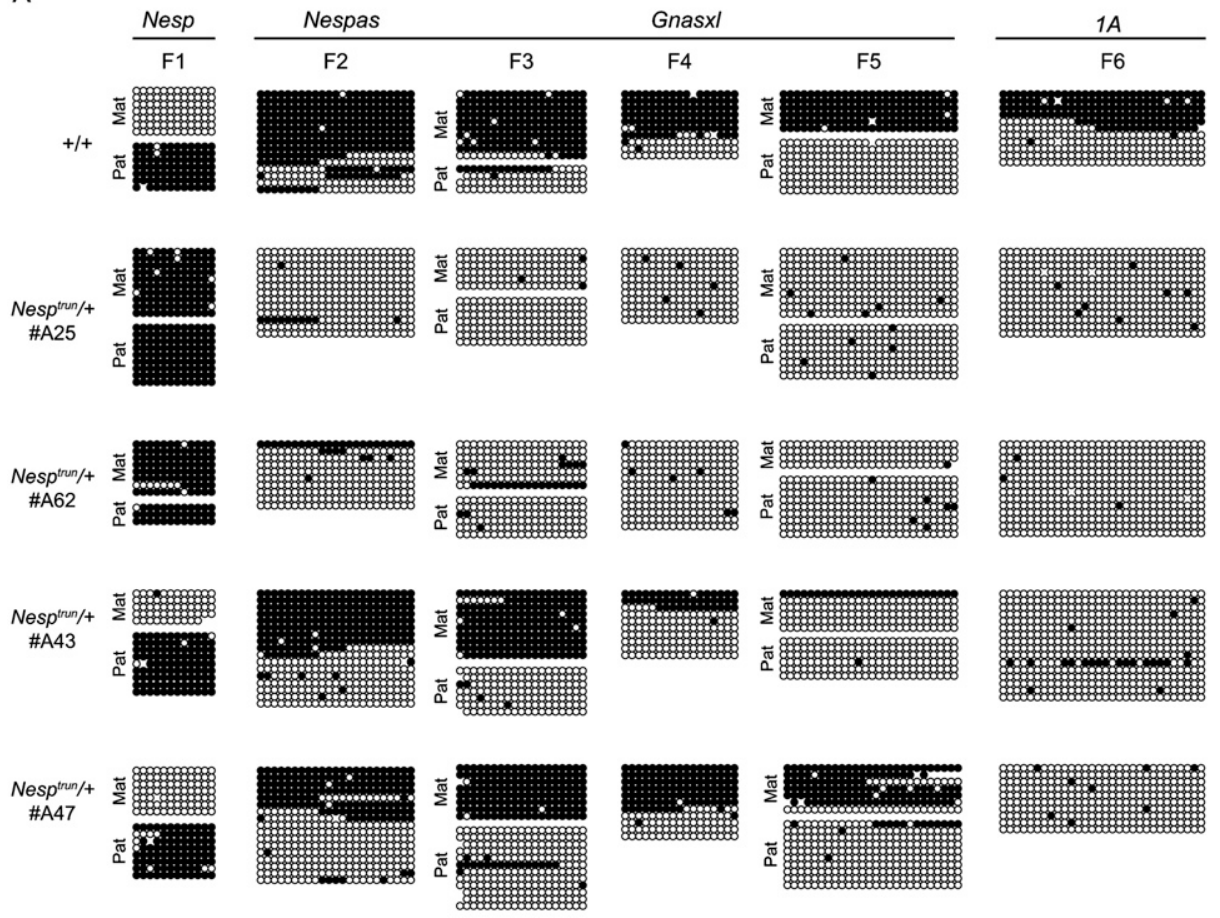

B

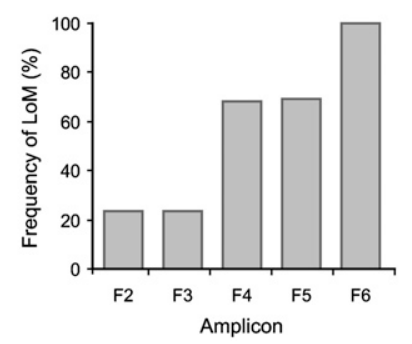

Figure 3. Maternal transmission of the Nesp truncation results in loss of methylation of maternal germline DMRs. $(A)$ Bisulphite sequence profiles from neonatal brain DNA from Nesp ${ }^{\text {trun/+ }}$ and wild-type $(+/+)$ pups. A total of 34 Nesp ${ }^{\text {trun/+ }}$ pups was analyzed by COBRA (see $B$ ), and sequences for one or more amplicon were obtained from seven mutants. Representative sequences from Nesp ${ }^{\text {trun } /+}$ mutants with complete LoM (\#A25, \#A62) or partial LoM (\#A43, \#A47) of the maternal germline DMRs are shown. Sequence polymorphisms allowed maternal and paternal alleles to be discriminated for products F1, F3, and F5. (B) Summary showing the frequency of LoM across the DMRs in neonatal brain in Nesp trun/+ mutants collected from 11 litters. The number of Nesp trun/+ pups typed by COBRA for amplicons F2, F3, and F6 was 34; for F4 was 22; and for F5 was 26; seven wild-type pups were similarly scored and found to have the normal pattern of DMRs. These data are a combination of COBRA results from crosses of Nesp ${ }^{\text {trun/+ }}$ females with

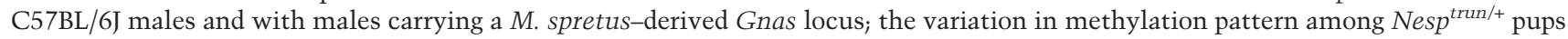
was similar in the two crosses. See Figure 1A for the location of the amplicons within the DMRs. 
A
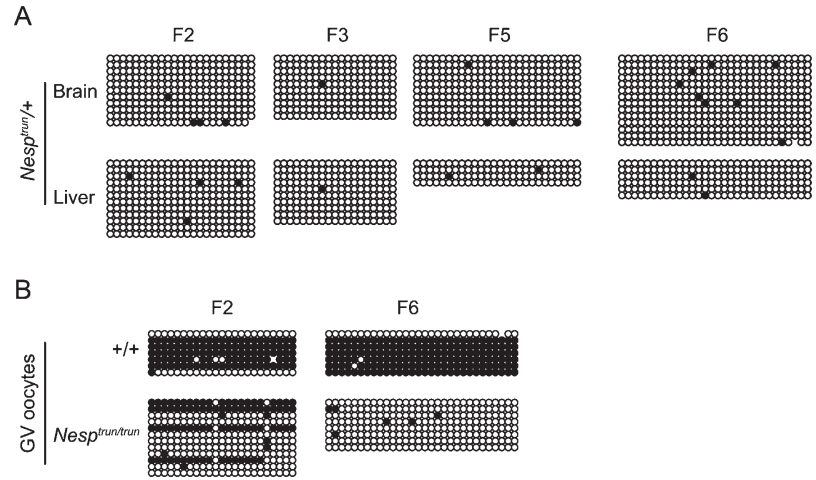

Figure 4. Loss of methylation occurs in oocytes of Nesp $\mathrm{prun} /+^{\text {t }}$ females. (A) Bisulphite sequences for the Nespas/Gnasxl (amplicons F2, F3, and F5) and 1A DMRs (F6) of brain and liver DNAs from the same pup (\#1D) inheriting the Nesp ${ }^{\text {trun }}$ allele maternally $\left(N e s p^{\text {trun } /+}\right)$. DNAs from four other pups from the same litter were assayed in both brain and liver by COBRA, revealing similar aberrant methylation profiles in the two tissues (Supplemental Fig. 4). (B) Bisulphite sequences for amplicons F2 and F6 obtained in GV oocytes from Nesp $p^{\text {trun }}$ homozygous females in comparison with wild type $(+/+)$. See Figure 1A for the location of the PCR products within the DMRs.

methylation of the DMRs (Supplemental Fig. 4). Because analysis of methylation in Nesp trun/+ pups was done after the first maternal transmission of the Nesp ${ }^{\text {trun }}$ allele, the variable methylation found is not a consequence of a failure to erase a pre-existing methylation pattern, as the Nespas/Gnasxl and 1A DMRs on the Nesp ${ }^{\text {trun }}$ allele will have been unmethylated in the transmitting females, being inherited as the unmethylated paternal allele. Moreover, the partial methylation present in a subset of Nesp $p^{\text {trun/+ }}$ mutants does not appear to be heritable, because analysis of primordial germ cells isolated from embryonic day 14.5 (E14.5) Nesp $p^{\text {trun } /+}$ female embryos showed that all DMRs were unmethylated (data not shown).

The pattern of LoM was found to be very similar in two neonatal tissues (brain and liver) from the same mutants (Fig. 4A; Supplemental Fig. 3). As brain represents a tissue with relatively abundant Nesp expression and liver represents a tissue in which expression is very low (data not shown), LoM occurs irrespective of the actual expression status of Nesp in somatic tissues. In addition, bisulphite sequence analysis indicated that when there was LoM at a given DMR, it was generally complete; i.e., there were few, if any, methylated maternal allele sequences or signs of mosaic methylation (Figs. 3A, 4A). Both observations suggested that LoM had occurred either in the female germline or in the early embryo. Because maternal transmission of the Nesp ${ }^{\text {trun }}$ allele was found to cause greatly reduced postnatal viability (as often occurs with altered imprinting of the Gnas locus) (Plagge and Kelsey 2006), very few homozygous Nesp ${ }^{\text {trun/trun }}$ adult females were available from which to recover oocytes. Nevertheless, in oocytes obtained from two surviving Nesp $p^{\text {trun/trun }}$ females, we detected complete LoM of the $1 \mathrm{~A}$ DMR but residual methylation of the Nespas/Gnasxl DMR (Fig. 4B). This confirmed a failure of methylation in the maternal germline, and also that the variable degree of LoM observed among Nesp ${ }^{\text {trun/+ }}$ pups most likely reflects differences among oocytes in the degree of LoM across the locus. (We cannot exclude that some modulation of methylation patterns may also occur after fertilization.) We do not know the reason for the partial establishment of methylation at the DMR closest to the $\beta$-globin cassette. The directionality of the partial methylation suggested that the cassette may not be fully effective at terminating transcription downstream from Nesp, so that if some run-through transcription occurs across a prospective DMR during oocyte growth, it might be sufficient to allow methylation, particularly those most proximal to the cassette. Although there was no evidence for run-through transcription in our $3^{\prime}$ RACE analysis (Fig. 2B), this technique may not be sufficiently sensitive to pick up longer, less abundant transcription products that might occur if transcription termination is not fully effective. Real-time PCR assays for primary transcripts at the Nespas/Gnasxl DMR in Nesp ${ }^{\text {trun/+ }}$ brain RNA did reveal the expected substantial down-regulation of transcription but were not sensitive enough to exclude the possibility of residual transcription downstream from the termination cassette (data not shown). Alternatively, there may be an effect on local chromatin organization downstream from transcription termination that permits some acquisition of methylation of the DMR sequences in closest proximity to the cassette.

Disrupted imprinted expression of Gnas locus transcripts as a result of loss of germline methylation

The LoM observed in Nesp $p^{\text {trun/+ }}$ pups is predicted to result in altered imprinting and expression of the Gnas locus transcripts, but the allelic expression profile should now depend on the precise methylation pattern in a given mutant. To investigate this, we typed mutants for methylation pattern by COBRA and separated them into three epigenotype classes based on the extent of LoM (Supplemental Fig. 5A). RNA was extracted from brain and brown adipose tissue, real-time RT-PCR used to quantify expression levels, and the Sequenom MassARRAY system used to determine allelic usage, based on a sequence polymorphism between C57BL/6 and spretus derived alleles in Gnas exon 11 that is common to all sense transcripts of the locus. Expression of the Nesp, Gnasxl, $1 A$, and Gnas transcripts was found to be altered largely as expected from the methylation profile in the three mutant epigenotype classes (Supplemental Fig. 5B,C). For example, LoM of the $1 A$ DMR was associated with derepression of the maternal allele of the $1 \mathrm{~A}$ transcript, and this was accompanied by down-regulation of the maternal allele of Gnas in brown adipose tissue but not in brain, consistent with the role of the $1 \mathrm{~A}$ DMR or transcript in tissue-specific imprinting of Gnas (Williamson et al. 2004; Liu et al. 2005). Nesp $p^{\text {trun/+ }}$ pups had anomalous phenotypes consistent with deregulated imprinting of the Gnas locus. A proportion of pups was noticeably heavier and edematous and had the broad-necked appearance 
(Supplemental Fig. 6A) typical of newborn mice that lack expression of maternal Gnas (Plagge and Kelsey 2006). On typing by COBRA, these pups were found to have LoM confined to the 1A DMR (Supplemental Fig. 6B), which causes reduced expression of maternal Gnas in tissues with imprinted expression (as in Supplemental Fig. 5). The remaining Nesp $p^{\text {trun/t }}$ pups were lighter at birth and were found to have more extensive LoM, giving upregulation of maternal Gnasxl transcripts as well as reduction in maternal Gnas. All epigenotype classes had very poor postnatal survival, with only a small proportion of the pups with LoM restricted to the $1 \mathrm{~A}$ DMR surviving to weaning; pups with more extensive LoM did not survive more than a few days after birth (Supplemental Fig. 6C).

Transcription across DMRs in oocytes is a common feature of maternally marked imprinted domains

At the Gnas locus, we show that transcription from Nesp is required for establishment of methylation of the maternal germline DMRs, but we predict that a similar mechanism operates at many, if not all, maternally marked imprinted loci. This is supported by the fact that most maternal germline DMRs are within transcription units and by the intronic location of imprinted retrotransposons (Wood et al. 2007). Indeed, 15 out of the 17 characterized maternal germline DMRs are in the introns of the genes they regulate (e.g., Igf2r) or, if at promoters, are downstream from alternative transcription start sites (e.g., Grb10) (Table 1; see below). In contrast, the three known paternal germline DMRs are intergenic, a location that might help preclude methylation in female gametes. To test our prediction, we surveyed several key imprinted loci, Grb10, Igf2r, Impact, Kcnq1, and Zac1, for transcripts crossing their germline DMRs in growing or mature metaphase II (MII) oocytes. In each case, tran- scripts were readily detected throughout oocyte growth and maturation (Fig. 5). Transcription across the Snrpn DMR in mouse oocytes has been described previously (Mapendano et al. 2006), and we were able to verify this in our own samples (data not shown). In order to ascertain whether transcription in oocytes was specific to maternal germline DMRs, we tested the H19DMR, which acquires methylation during spermatogenesis. Although this DMR is outside of the $H 19$ transcription unit, transcripts for the DMR have been described in somatic cells (Schoenfelder et al. 2007). We were readily able to detect these transcripts in RNA from E13.5 embryos, but not in growing or MII oocytes (Fig. $5 \mathrm{~B}$ ). We also tested whether the intragenic CGIs of nonimprinted loci were transcribed. For this, we selected four loci with intragenic CGIs for which methylation status is known (Song et al. 2005). For only one of the four genes were transcripts crossing the intragenic CGIs detected in growing or MII oocytes (Fig. 5C).

We were particularly interested to define the start sites for the transcripts present in oocytes, given that we found evidence for alternative start sites for Nesp (Supplemental Fig. 1), and we wished to see whether for promoter associated DMRs, alternative upstream start sites could be found that would provide transcription through the DMRs in oocytes. For each gene, detailed analysis by 5'RACE (in d10 and/or MII oocytes) identified one or more start sites in oocytes, usually distinct from the major sites used in embryos, and these were confirmed in separate RT-PCR experiments across all oocyte stages (summarized in Fig. 5A). At Zac1, for example, we found an alternative start site $\sim 30 \mathrm{~kb}$ upstream of the DMR that contains the promoter used predominantly in somatic tissues. The upstream start site accounts for the transcripts traversing the DMR and the vast majority of Zac1 transcripts in growing oocytes; transcripts from the

Table 1. Locations of germline DMRs in imprinted loci

\begin{tabular}{|c|c|c|c|c|}
\hline Locus (DMR) & Chromosome & DMR methylation & DMR location & Reference \\
\hline Gnas (Nespas/Gnasxl) & 2 & Maternal & Intron & Coombes et al. 2003 \\
\hline Gnas (1A) & 2 & Maternal & Intron & Liu et al. 2000 \\
\hline Mcts2 & 2 & Maternal & Intron & Wood et al. 2007 \\
\hline Peg10 & 6 & Maternal & Promoter & Ono et al. 2003 \\
\hline Peg1 & 6 & Maternal & Intron $^{\mathrm{a}}$ & Lucifero et al. 2002 \\
\hline Nap115 & 6 & Maternal & Intron & Wood et al. 2007 \\
\hline Peg3 & 7 & Maternal & Intron & Kim et al. 2003 \\
\hline Snrpn & 7 & Maternal & Intron & Mapendano et al. 2006 \\
\hline Kcnq1 (KvDMR) & 7 & Maternal & Intron & Yatsuki et al. 2002 \\
\hline Inpp $5 f$ & 7 & Maternal & Intron & Wood et al. 2007 \\
\hline Zac1 & 10 & Maternal & Intron & This study \\
\hline Grb10 & 11 & Maternal & Intron & Arnaud et al. 2003 \\
\hline U2af1-rs1 & 11 & Maternal & Intron & Wood et al. 2007 \\
\hline Peg13 & 15 & Maternal & Intron & Ruf et al. 2007 \\
\hline Slc38a4 & 15 & Maternal & Promoter & G. Kelsey (unpubl.) \\
\hline Igf2r (Air) & 17 & Maternal & Intron & Stoger et al. 1993 \\
\hline Impact & 18 & Maternal & Intron & Okamura et al. 2000 \\
\hline H19 & 7 & Paternal & Intergenic & Tremblay et al. 1997 \\
\hline Rasgrf1 & 9 & Paternal & Intergenic & Shibata et al. 1998 \\
\hline Dlk1-Gt12 (IG-DMR) & 12 & Paternal & Intergenic & Takada et al. 2002 \\
\hline
\end{tabular}

${ }^{\mathrm{a} E v i d e n c e ~ f o r ~ u p s t r e a m ~ s t a r t ~ s i t e s ~ f r o m ~ E S T s . ~}$ 
Chotalia et al.

Figure 5. Transcription across maternal germline DMRs in oocytes is common among imprinted genes. (A) On the left, schemes representing the imprinted loci analyzed, Igf2r, Grb10, Kcnq1, Zac1, and Impact, showing the locations of the primers used for RT-PCRs in relation to the germline DMRs (filled circles). The schemes are not to scale, and for simplicity, not all exons of the genes are shown. Characterized start sites or start sites determined by 5'RACE analysis are indicated by arrows, with those above the line representing start sites detected in somatic cells and those below the line in oocytes. Novel exons identified by 5 'RACE are shown as gray boxes. On the right, RT-PCRs for these loci for transcripts traversing the DMRs in day 5, day 10, day 15 and MII oocytes are shown. For both Igf2r and Grb10, RTPCRs labeled with Ex1-F/Ex3-R1 assay transcripts from the canonical somatic promoter and RT-PCRs labeled with Un1-F/Ex3-R2 assay the novel start sites identified in oocytes by 5'RACE. The multiple bands for Zac1 reflect alternative splicing of the various $5^{\prime}$ untranslated exons, exon 8 being the first coding exon. (B) RT-PCR analysis of transcripts for the H19 paternal germline DMR. The RT-PCR represents amplicon 4 from Schoenfelder et al. (2007). (C) RT-PCR analysis of transcripts crossing the intragenic CGIs of four nonimprinted genes. Gene Sp6 represents locus PvuII 44, Chst8 is PstI 53, Sema6c is PstI 58, and $A d r a 1 b$ is PvuII 66 from Song et al. (2005). In the schemes to the left, the locations of the CGIs are represented by the open circles. In each panel, the lanes marked Co represent control amplification from E13.5 embryo RNA, and $\mathrm{H}$ indicates no template control. The left lanes on each gel show a 100-bp marker ladder, with size marker relevant to the RT-PCR product indicated by arrows.
A
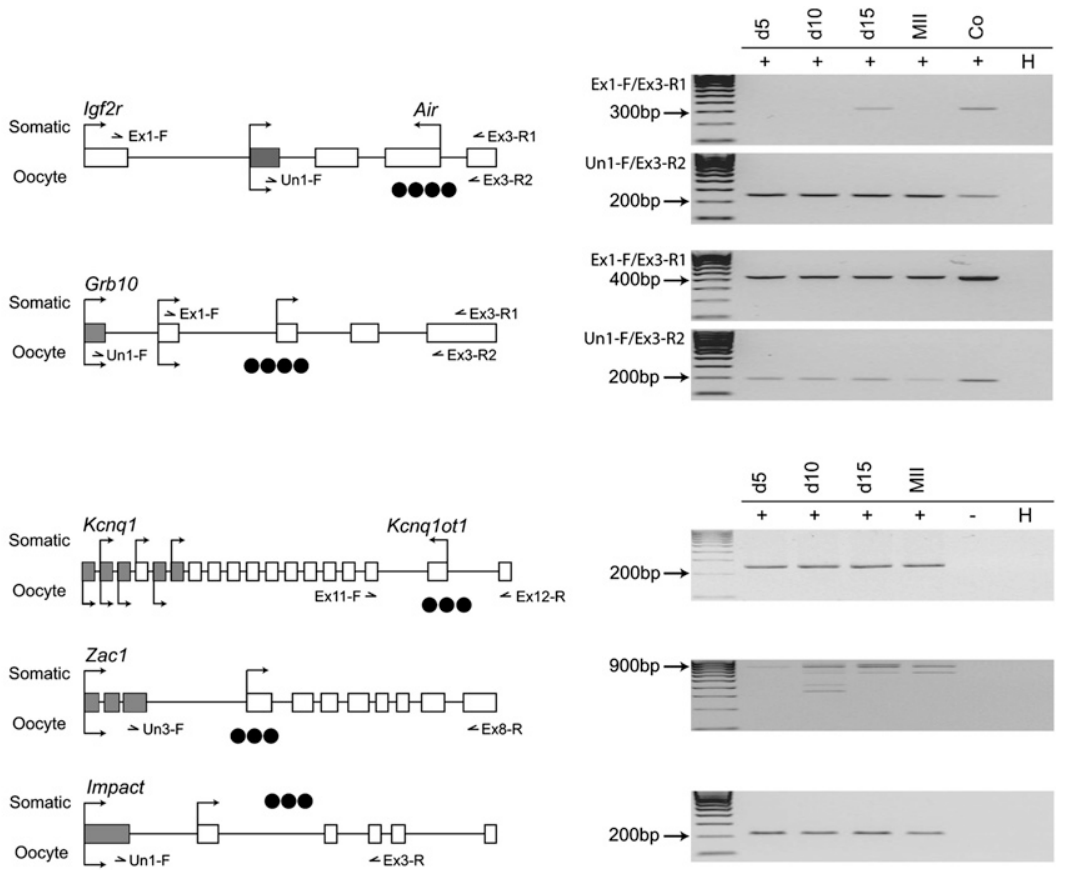

B
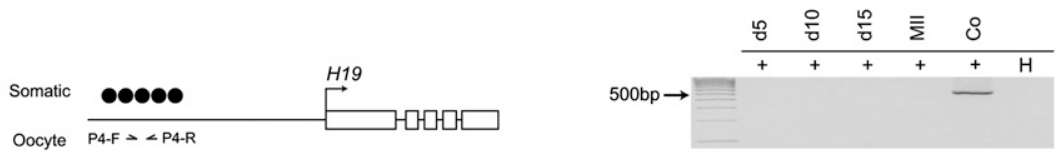

C
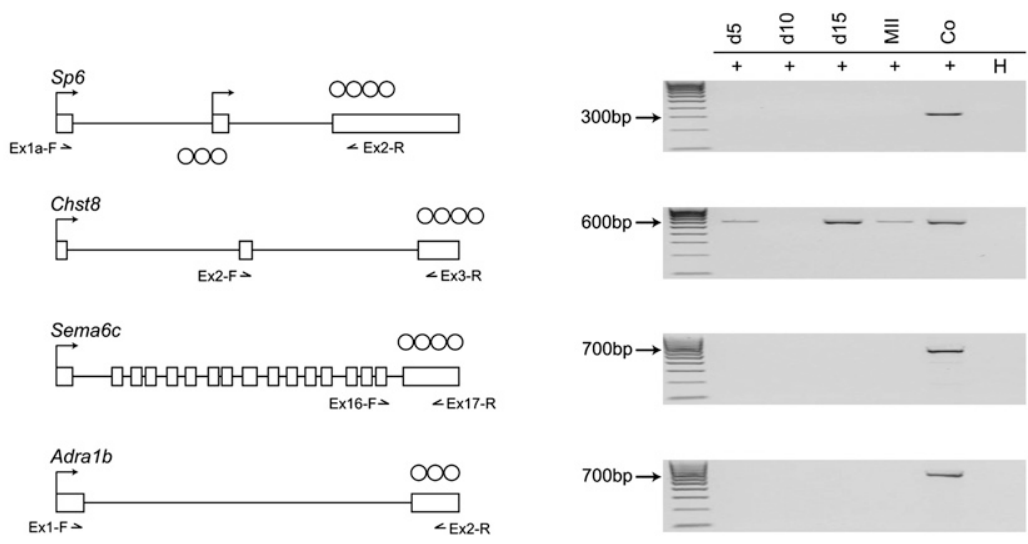
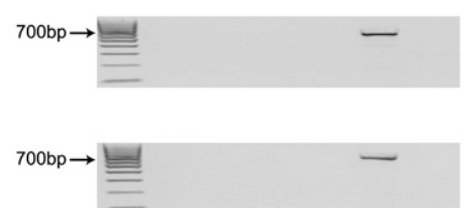

DMR promoter were only weakly detected in growing oocytes. Perhaps surprisingly, even for many of the genes for which the DMR is in an intron of the characterized transcription unit (e.g., Grb10, Igf2r, Kcnq1), alternative start sites were identified by 5'RACE in oocytes. At Igf2r, a targeted deletion of the well-defined somatic promoter did not disrupt methylation of the Air germline DMR (Sleutels et al. 2003). However, transcripts initiating from this promoter were not consistently detected in oocytes, instead $I g f 2 r$ was prominently expressed from transcription start sites located in intron 1 (Fig. 5A). This might explain why the promoter deletion does not impair imprinting of the locus. (It should be noted that the additional start sites for these transcripts may not be oocyte-specific, as in many cases they could be detected weakly by RT-PCR in embryo or somatic tissues; conversely, the predominant somatic start site for Grb10 was also readily detected in oocytes [Fig. 5A].)

In conclusion, the frequent location of maternal germline DMRs within transcription units and the fact that transcripts traversing these DMRs are detected in growing oocytes suggest that transcription is a common requirement for de novo methylation of maternal imprint marks.

\section{Discussion}

In this study, we provide evidence that transcriptional events are required for the establishment of DNA methylation imprints in the female germline. Functional 
evidence in support of this concept was provided by truncating the Nesp transcript at the Gnas locus, which resulted in LoM of the maternal germline DMRs. LoM of the $1 A$ DMR was fully penetrant on maternal transmission of the truncation allele, as well as in oocytes, but there was variable LoM of the Nespas/Gnasxl DMR. In humans, deletions of the NESP55 region also result in LoM of the GNAS DMRs on the maternal allele (Bastepe et al. 2005). We also suggest that transcription is a common requirement for maternal germline DMRs, because most such DMRs are located within transcription units, and where this is apparently not the case, further examination provided evidence for alternative upstream start sites used in oocytes. Furthermore, for all eight maternal germline DMRs we tested, transcripts traversing the prospective DMRs were detected in growing oocytes. In contrast, this was not the case for the H19 DMR, which is methylated in the male germline, and intragenic CGIs of nonimprinted loci were also not consistently transcribed in oocytes and serve here as biologically appropriate controls.

\section{Possible mechanistic links between transcription and} DNA methylation establishment

What is the role of transcription and how can it be incorporated into a model that accounts for the normal methylation pattern of Gnas and other imprinted loci? We expect that the logic that explains imprinted methylation at Gnas will apply to all maternally marked imprinted domains. Based on the interaction of Dnmt3L with histone H3 (Ooi et al. 2007), we anticipate that the prospective DMRs at Nespas/Gnasxl and 1A have low levels of methylated H3K4 in growing oocytes, which could reflect relative inactivity of the associated promoters in growing oocytes. Consistent with this possibility, we found that Nespas, Gnasxl, and 1A transcripts were not readily detected during oocyte growth. The same model might apply to the Zac1 promoter DMR. We suggest that if prospective DMRs are inactive promoters with unmethylated H3K4, then they are likely to be packaged into relatively condensed chromatin, so transcription from upstream promoters may be necessary to create or maintain an open chromatin environment that allows Dnmt3a/Dnmt3L access to its preferred targets. Transcription might also play a more active role. There could be a direct interaction between nascent transcripts or the transcription complex and the de novo methylation machinery. Alternatively, transcription could recruit factors, such as LSD1 or SMCX (Shi et al. 2004; Iwase et al. 2007), necessary for demethylating H3K4 at the DMRs. Future experiments will seek to address the temporal relationship between ongoing transcription and de novo methylation.

It is important to highlight in our model that none of the factors implicated in DMR methylation-CpG periodicity, promoter activity/H3K4 methylation status, and transcription-is sufficient by itself. Rather, we expect that all have to apply in order for a DMR to become methylated. This is illustrated, for example, by the Gnas
CGI. It has a CpG periodicity apparently similar to the Nespas/Gnasxl and 1A DMRs (data not shown), and transcripts initiating at Nesp in oocytes traverse the CGI just as they do these DMRs. In this case, it might be that the methylation state of H3K4 at the Gnas CGI is inappropriate for Dnmt3L engagement, and the fact that Gnas transcripts are readily detected in oocytes suggests that the Gnas CGI houses an active promoter marked by H3K4me3 and protected from DNA methylation. Similarly, although intragenic CGIs of some nonimprinted loci may be transcribed in oocytes, without the appropriate $\mathrm{CpG}$ periodicity or $\mathrm{H} 3 \mathrm{~K} 4$ methylation state they would not be targets for Dnmt3a/Dnmt3L.

\section{Oocyte transcription units and the evolution of imprinted loci}

It is notable that in all the cases we studied, the transcripts traversing the DMRs and that we propose are involved in methylation establishment are protein-coding transcripts, and this sets them apart from the noncoding antisense transcripts, such as Air, Kcnq1ot1, and Nespas, which are involved in the silencing and methylation of imprinted promoters in somatic tissues (Pauler et al. 2007). This suggests that they encode proteins with functions in the oocyte, or the preimplantation embryo, and the activity of these transcription units therefore may have provided the opportunity for imprinting to arise. It has been proposed that imprinted domains could evolve through insertion of CpG-rich elements, such as retroposons (Wood et al. 2007), into transcription units active in oocytes, and this is fully consistent with our model. Such events could be the origin of DMRs such as Air and KVDMR1, which became the promoters for long, noncoding RNAs that subsequently came to orchestrate domain-wide monoallelic expression.

In many cases, we detected alternative promoters for transcripts in oocytes distinct from the major promoters used in somatic tissues (which, generally, did not alter their coding potential). This suggests a new possibility for the evolution of imprinting, whereby recruitment of oocyte-expressed promoters upstream of susceptible CGIs could give rise to new DMRs and establish novel imprinted domains. This might explain loci such as Grb10, Snrpn, and Zac1 that are characterized by multiple promoters for transcripts with the same coding potential. Consistent with this possibility, while the Gnas locus is highly conserved, we did not find evidence for a Nesp homolog in noneutherian mammals. In marsupials, the Gnas locus does contain a Gnasxl homolog, but neither the Gnasxl CGI nor any other CGI in the locus is a DMR (G. Kelsey and E. Ivanova, unpubl.). It is possible that the oocyte promoters of these "imprinting" transcripts represent a specific subset of promoters with shared, specific transcription factors and that subsequently have been selected especially for their ability to imprint genes.

\section{Implications for imprinted gene disorders}

Our model also has important implications for the nature of genetic defects giving rise to imprinting disorders in 
humans. It offers an explanation for how microdeletions disrupt imprinting of GNAS in PHP1b (Bastepe et al. 2003, 2005) or cause LoM of the SNRPN DMR in AS (Buiting et al. 1995; Dittrich et al. 1996), and this should encourage a search for similar lesions in other imprinted disorders. SNRPN is characterized as having multiple, alternative upstream start sites (Buiting et al. 1995; Dittrich et al. 1996), so the AS microdeletions could involve essential oocyte promoters or enhancers or otherwise prevent germline transcription across the SNRPN DMR. In mouse oocytes deficient in the KRAB finger protein $\mathrm{Zfp} 57$, the Snrpn DMR is unmethylated (Li et al. 2008), and it will be interesting to examine whether this factor is involved in regulating transcription events across the Snrpn DMR. It is interesting to note that an alternative upstream promoter has been described for human ZAC1 (Valleley et al. 2007), similar to that we describe here for mouse Zac1, and such elements become candidate regions to analyze in other imprinted disorders. It is also possible that defects affecting multiple imprinting loci, such as cases of maternal hypomethylation syndrome (Mackay et al. 2006), could arise because of mutations within transcription factors required for the expression of subsets of the oocyte transcripts implicated here in imprint establishment. Finally, differences in the timing of the transcription events through DMRs could explain why imprinting of the various domains appears to be established at different times during oocyte growth (Obata and Kono 2002; Lucifero et al. 2004; Hiura et al. 2006).

\section{Materials and methods}

\section{Isolation of growing and mature oocytes}

Growing oocytes were collected from C57BL/6J mice (Charles River Laboratories) at 5, 10, and 15 dpp. Ovaries from two to three mice were dissected in PBS $(\mathrm{pH} 7.2)$ and digested for 10$15 \mathrm{~min}$ at $37^{\circ} \mathrm{C}$ in PBS containing $2 \mathrm{mg} / \mathrm{mL}$ collagenase, $0.025 \%$ trypsin, and $0.02 \mathrm{mg} / \mathrm{mL}$ DNase. The digestion mixture was diluted by an equal volume of HEPES buffered M2 medium and oocyte-cumulus cell aggregates dissociated by mechanical disruption in a finely drawn pipette. Oocytes were identified as the larger, refractile cells and washed free of somatic cells by transfer through two to three dishes of M2. GV oocytes were collected at 42-h post-injection with 7.5 IU pregnant mare's serum gonadotropin (PMS). Ovaries were dissected in PBS, oocyte-cumulus complexes released by puncturing large follicles, and cumulus cells removed by digestion as above. Only oocytes with an intact GV and no apparent degeneration were collected. MII oocytes were collected from 7-wk-old superovulated females as described previously (Coombes et al. 2003).

\section{Expression analysis}

Total RNA from 65-200 oocytes was extracted by the TRIzol method (Invitrogen) and DNase treated using Turbo DNase I (Ambion). cDNA was synthesized using Superscript III (Invitrogen) and random hexamer primers. Duplicate sets of samples were produced with RT omitted to detect amplification from contaminating DNA. PCR amplification was done with HotStarTaq DNA polymerase (Qiagen) in 20- to $25-\mu \mathrm{L}$ volumes using $1-2 \mu \mathrm{L}$ of the RT reaction under standard PCR conditions. 5' RACE assays were conducted using the RLM-RACE kit
(Ambion), using RNA from $\sim 100$ oocytes (10 dpp), or from 118 MII oocytes with $1 \mu \mathrm{g}$ E4 chick RNA as carrier. A 3'RACE assay for Nesp transcripts was conducted by priming total RNA from 23-75 MII oocytes with an oligo dT primer (Invitrogen GeneRacer kit). For both RACE assays, treated RNA was reverse transcribed using Superscript III, and nested PCRs were conducted with approximately one to eight oocyte equivalents per first-round PCR. Transcripts detected by RT-PCR and RACE were verified by sequencing after TOPO TA cloning (Invitrogen). Primer information for RT-PCR and RACE assays is presented in Supplemental Table 1 .

\section{Methylation analysis}

Analysis of DMR methylation by bisulphite modification was done essentially as described previously (Ruf et al. 2007). For neonatal tissues (brain and liver) from Nesp ${ }^{\text {trun } /+}$ and Nesp ${ }^{+/+}$ mice, DNA was extracted using the Allprep DNA/RNA minikit (Qiagen), $1 \mu \mathrm{g}$ of DNA samples treated using the EpiTect kit (Qiagen), and the equivalent of $125 \mathrm{ng}$ of input DNA used per PCR. Growing oocytes (250-500) and GV oocyes (80-110) were lysed directly in $20-40 \mu \mathrm{L}$ PBS containing $20 \mu \mathrm{g} / \mathrm{mL}$ proteinase $\mathrm{K}$ and $40 \mathrm{mM}$ SDS, and $20 \mu \mathrm{L}$ of these lysates treated using the Imprint DNA Modification kit (Sigma). Converted DNA from MII oocytes was prepared previously (Ruf et al. 2007). PCRs from bisulphite-treated oocyte DNA contained the equivalent of 2035 oocytes. For growing oocyte DNAs, the results shown were obtained from two independent bisulphite treatments performed on a batch of oocytes collected at the same time; for GV oocytes, the results are from single treatments of 80-110 oocytes. Amplification, COBRA (using MseI or TaqI), and cloning and sequencing of PCR products were performed as previously described (Ruf et al. 2007). Bisulphite sequences were analyzed using BiQ Analyser (Bock et al. 2005), with a conversion frequency cut-off of $88 \%$ and additional sequences with identical patterns of unconverted cytosines (outside of CpGs) discarded as possible clonal amplification products. Primer information is presented in Supplemental Table 2.

\section{Generation of the Nesp ${ }^{\text {trun }}$ targeted allele}

The targeting construct was prepared using homologous recombination in yeast, as described previously (Plagge et al. 2005), and inserted a 1.2-kb fragment from the rabbit $\beta$-globin gene (kindly provided by Dr. Denise Barlow) (Sleutels et al. 2002); nucleotides 31,392-32,590 in M18818) downstream from Nesp exon 2 (position 141,627 in AL593857.10) in the correct orientation to truncate Nesp transcripts. The construct also contained a selection cassette with URA3 (for selection in yeast) and neo ${ }^{r}$ (for selection in embryonic stem [ES] cells) flanked by loxP sites. The targeting construct was linearized with NotI and electroporated into E14 129/Ola ES cells. Colonies surviving G418 selection were screened by Southern blot analysis of AflII digested DNA with a 1.5-kb fragment (nucleotides 134,606-136,087), which resides $5^{\prime}$ to the insertion of the targeting construct, and XhoI digested DNA with a 601-bp fragment (nucleotides 147,539148,139 ) at the $3^{\prime}$ end. Chimeric mice were generated by injecting targeted ES cells into C57BL/6J blastocysts; germline transmission of the targeted allele was confirmed by PCR of tail lysate DNA; and transmitting male chimaeras were crossed with homozygous female Cre-deletor mice (Schwenk et al. 1995) for ubiquitous excision of the selection cassette. The amplification product specific for the excised allele was cloned and sequenced to confirm the correct Cre recombination event. Nesp $p^{\text {trun }}$ heterozygotes were maintained on a C57BL/6J background by paternal transmission of the Nesp ${ }^{\text {trun }}$ allele, owing to the high rate of 
lethality of Nesp ${ }^{\text {trun/+ }}$ pups after maternal transmission. Experimental mice were obtained from maternal transmission of the Nesp ${ }^{\text {trun }}$ allele crossed with C57BL/6J males or (C57BL/6JxDS2) males (DS2 is a strain that carries the Gnas region of chromosome 2 from Mus spretus on an F1/C57BL/6)xCBA/Ca) background) (Plagge et al. 2005). All analysis of Nesp ${ }^{\text {trun } /+}$ pups was done after the first maternal transmission of the Nesp trun allele.

\section{GenBank accession numbers}

Nucleotide numbers for the Gnas locus referred to in the text correspond to sequence accession number AL593857.10. Accession numbers for the novel exons and start sites identified in oocyte and embryo RNA by 5'RACE and RT-PCR for the imprinted loci shown in Figure 5 are listed in Supplemental Table 3.

\section{Acknowledgments}

We are grateful to Denise Barlow for providing the rabbit $\beta$-globin transcription termination cassette. We thank the Gene Targeting Facility at the Babraham Institute for generating the knockout mice, members of the Small Animal Facilities for their conscientious mouse husbandry, Anne Segonds-Pichon for assistance with statistical analysis, and Simon Andrews for bioinformatics support. We are very grateful to Jo Peters, Wolf Reik, and Rebecca Oakey for thoughtful discussions and comments on the manuscript. This work was supported by the Biotechnology and Biological Sciences Research Council of the United Kingdom and the European Union (STREP Savebeta contract 036903 in the Framework Programme 6 [FP6] of the European Community). N.R. was sponsored by a post-doctoral fellowship from the German Academic Exchange Service (DAAD) and a Marie-Curie Intra European Fellowship from the European Community (FP7) 2007-2013) under grant agreement number PIEF-GA-2008220912. K.J. was supported by a Genes \& Development Summer Studentship from the Genetics Society.

\section{References}

Arnaud, P., Monk, D., Hitchins, M., Gordon, E., Dean, W., Beechey, CV., Peters, J., Craigen, W., Preece, M., and Stanier, P., et al. 2003. Conserved methylation imprints in the human and mouse GRB10 genes with divergent allelic expression suggests differential reading of the same mark. Hum. Mol. Genet. 12: 1005-1019.

Arnaud, P., Hata, K., Kaneda, M., Li, E., Sasaki, H., Feil, R., and Kelsey, G., 2006. Stochastic imprinting in the progeny of Dnmt3L $\mathrm{L}^{-1-}$ females. Hum. Mol. Genet. 15: 589-598.

Bastepe, M., Frohlich, L.F., Hendy, G.N., Indridason, O.S., Josse, R.G., Koshiyama, H., Korkko, J., Nakamoto, J.M., Rosenbloom, A.L., and Slyper, A.H., et al. 2003 Autosomal dominant pseudohypoparathyroidism type $\mathrm{Ib}$ is associated with a heterozygous microdeletion that likely disrupts a putative imprinting control element of GNAS. I. Clin. Invest. 112: 1255-1263.

Bastepe, M., Frohlich, L.F., Linglart, A., Abu-Zahra, H.S., Tojo, K., Ward, L.M., and Juppner, H., 2005. Deletion of the NESP55 differentially methylated region causes loss of maternal GNAS imprints and pseudohypoparathyroidism type Ib. Nat. Genet. 37: 25-27.

Bernstein, B.E., Kamal, M., Lindblad-Toh, K., Bekiranov, S., Bailey, D.K., Huebert, D.J., McMahon, S., Karlsson, E.K., Kulbokas III, E.J., and Gingeras, T.R., et al. 2005. Genomic maps and comparative analysis of histone modifications in human and mouse. Cell 120: 169-181.

Bock, C., Reither, S., Mikeska, T., Paulsen, M., Walter, J., and Lengauer, T., 2005. BiQ Analyzer: Visualization and quality control for DNA methylation data from bisulfite sequencing. Bioinformatics 21: 4067-4068.

Bourc'his, D., Xu, G.L., Lin, C.S., Bollman, B., and Bestor, T.H., 2001. Dnmt3L and the establishment of maternal genomic imprints. Science 294: 2536-2539.

Buiting, K., Saitoh, S., Gross, S., Dittrich, B., Schwartz, S., Nicholls, R.D., and Horsthemke, B., 1995. Inherited microdeletions in the Angelman and Prader-Willi syndromes define an imprinting centre on human chromosome 15. Nat. Genet. 9: 395-400.

Buiting, K., Lich, C., Cottrell, S., Barnicoat, A., and Horsthemke, B., 1999. A 5-kb imprinting center deletion in a family with Angelman syndrome reduces the shortest region of deletion overlap to 880 bp. Hum. Genet. 105: 665-666.

Coombes, C., Arnaud, P., Gordon, E., Dean, W., Coar, E.A., Williamson, C.M., Feil, R., Peters, J., and Kelsey, G., 2003. Epigenetic properties and identification of an imprint mark in the Nesp-Gnasxl domain of the mouse Gnas imprinted locus. Mol. Cell. Biol. 23: 5475-5488.

Dittrich, B., Buiting, K., Korn, B., Rickard, S., Buxton, J., Saitoh, S., Nicholls, R.D., Poustka, A., Winterpacht, A., Zabel, B., et al. 1996. Imprint switching on human chromosome 15 may involve alternative transcripts of the SNRPN gene. Nat. Genet. 14: 163-170.

Ferguson-Smith, A.C. and Greally, J.M. 2007. Epigenetics: Perceptive enzymes. Nature 449: 148-149.

Hata, K., Okano, M., Lei, H., and Li, E., 2002. Dnmt3L cooperates with the Dnmt3 family of de novo DNA methyltransferases to establish maternal imprints in mice. Development 129: 1983-1993.

Hiura, H., Obata, Y., Komiyama, J., Shirai, M., and Kono, T., 2006. Oocyte growth-dependent progression of maternal imprinting in mice. Genes Cells 11: 353-361.

Hutter, B., Helms, V., and Paulsen, M., 2006. Tandem repeats in the CpG islands of imprinted genes. Genomics 88: 323 332.

Illingworth, R., Kerr, A., Desousa, D., Jorgensen, H., Ellis, P., Stalker, J., Jackson, D., Clee, C., Plumb, R., Rogers, J., et al. 2008. A novel CpG island set identifies tissue-specific methylation at developmental gene loci. PLoS Biol. 6: e22. doi: 10.1371/journal.pbio.0060022.

Iwase, S., Lan, F., Bayliss, P., de la Torre-Ubieta, L., Huarte, M., Qi, H.H., Whetstine, J.R., Bonni, A., Roberts, T.M., and Shi, Y., 2007. The X-linked mental retardation gene SMCX/ JARID1C defines a family of histone H3 lysine 4 demethylases. Cell 128: 1077-1088.

Jia, D., Jurkowska, R.Z., Zhang, X., Jeltsch, A., and Cheng, X., 2007. Structure of Dnmt3a bound to Dnmt3L suggests a model for de novo DNA methylation. Nature 449: 248-251.

Jurkowska, R.Z., Anspach, N., Urbanke, C., Jia, D., Reinhardt, R., Nellen, W., Cheng, X., and Jeltsch, A., 2008. Formation of nucleoprotein filaments by mammalian DNA methyltransferase Dnmt3a in complex with regulator Dnmt3L. Nucleic Acids Res. 36: 6656-6663. doi: 10.1093/nar/gkn747.

Kaneda, M., Okano, M., Hata, K., Sado, T., Tsujimoto, N., Li, E., and Sasaki, H., 2004. Essential role for de novo DNA methyltransferase Dnmt3a in paternal and maternal imprinting. Nature 429: 900-903.

Kim, J., Kollhoff, A., Bergmann, A., and Stubbs, L., 2003. Methylation-sensitive binding of transcription factor YY1 to an insulator sequence within the paternally expressed imprinted gene, Peg3. Hum. Mol. Genet. 12: 233-245.

Kim, T.H., Barrera, L.O., Zheng, M., Qu, C., Singer, M.A., Richmond, T.A., Wu, Y., Green, R.D., and Ren, B., 2005. A high-resolution map of active promoters in the human genome. Nature 436: 876-880. 
Li, X., Ito, M., Zhou, F., Youngson, N., Zuo, X., Leder, P., and Ferguson-Smith, A.C., 2008. A maternal-zygotic effect gene, Zfp57, maintains both maternal and paternal imprints. Dev. Cell 15: 547-557.

Liu, J., Yu, S., Litman, D., Chen, W., and Weinstein, L.S., 2000. Identification of a methylation imprint mark within the mouse Gnas locus. Mol. Cell. Biol. 20: 5808-5817.

Liu, J., Chen, M., Deng, C., Bourc'his, D., Nealon, J.G., Erlichman, B., Bestor, T.H., and Weinstein, L.S., 2005. Identification of the control region for tissue-specific imprinting of the stimulatory G protein $\alpha$-subunit. Proc. Natl. Acad. Sci. 102: 5513-5518.

Lucifero, D., Mertineit, C., Clarke, H.J., Bestor, T.H., and Trasler, J.M., 2002. Methylation dynamics of imprinted genes in mouse germ cells. Genomics 79: 530-538.

Lucifero, D., Mann, M.R., Bartolomei, M.S., and Trasler, J.M., 2004. Gene-specific timing and epigenetic memory in oocyte imprinting. Hum. Mol. Genet. 13: 839-849.

Mackay, D.J., Boonen, S.E., Clayton-Smith, J., Goodship, J., Hahnemann, J.M., Kant, S.G., Njolstad, P.R., Robin, N.H., Robinson, D.O., and Siebert, R., et al. 2006. A maternal hypomethylation syndrome presenting as transient neonatal diabetes mellitus. Hum. Genet. 120: 262-269.

Mapendano, C.K., Kishino, T., Miyazaki, K., Kondo, S., Yoshiura, K., Hishikawa, Y., Koji, T., Niikawa, N., and Ohta, T., 2006. Expression of the Snurf-Snrpn IC transcript in the oocyte and its putative role in the imprinting establishment of the mouse 7C imprinting domain. J. Hum. Genet. 51: 236-243.

Neumann, B., Kubicka, P., and Barlow, D.P., 1995. Characteristics of imprinted genes. Nat. Genet. 9: 12-13.

Obata, Y., and Kono, T. 2002. Maternal primary imprinting is established at a specific time for each gene throughout oocyte growth. J. Biol. Chem. 277: 5285-5289.

Okamura, K., Hagiwara-Takeuchi, Y., Li, T., Vu, T.H., Hirai, M., Hattori, M., Sakaki, Y., Hoffman, A.R., and Ito, T., 2000. Comparative genome analysis of the mouse imprinted gene impact and its nonimprinted human homolog IMPACT: Toward the structural basis for species-specific imprinting. Genome Res. 10: 1878-1889.

Ono, R., Shiura, H., Aburatani, H., Kohda, T., Kaneko-Ishino, T., and Ishino, F., 2003. Identification of a large novel imprinted gene cluster on mouse proximal chromosome 6. Genome Res. 13: 1696-1705.

Ooi, S.K., Qiu, C., Bernstein, E., Li, K., Jia, D., Yang, Z., Erdjument-Bromage, H., Tempst, P., Lin, S.P., Allis, C.D., Allis, C.D. et al. 2007. DNMT3L connects unmethylated lysine 4 of histone $\mathrm{H} 3$ to de novo methylation of DNA. Nature 448: 714-717.

Pauler, F.M., Koerner, M.V., and Barlow, D.P., 2007. Silencing by imprinted noncoding RNAs: Is transcription the answer? Trends Genet. 23: 284-292.

Plagge, A., and Kelsey, G. 2006. Imprinting the Gnas locus. Cytogenet. Genome Res. 113: 178-187.

Plagge, A., Isles, A.R., Gordon, E., Humby, T., Dean, W., Gritsch, S., Fischer-Colbrie, R., Wilkinson, L.S., and Kelsey, G., 2005 Imprinted Nesp55 influences behavioral reactivity to novel environments. Mol. Cell. Biol. 25: 3019-3026.

Reik, W., and Walter, J. 2001a. Genomic imprinting: Parental influence on the genome. Nat. Rev. Genet. 2: 21-32.

Reik, W., and Walter, J. 2001b. Evolution of imprinting mechanisms: The battle of the sexes begins in the zygote. Nat. Genet. 27: 255-256.

Reinhart, B., Paoloni-Giacobino, A., and Chaillet, J.R., 2006. Specific differentially methylated domain sequences direct the maintenance of methylation at imprinted genes. Mol. Cell. Biol. 26: 8347-8356.
Ruf, N., Bahring, S., Galetzka, D., Pliushch, G., Luft, F.C., Nurnberg, P., Haaf, T., Kelsey, G., and Zechner, U., 2007. Sequence-based bioinformatic prediction and QUASEP identify genomic imprinting of the KCNK9 potassium channel gene in mouse and human. Hum. Mol. Genet. 16: 2591-2599.

Schoenfelder, S., Smits, G., Fraser, P., Reik, W., and Paro, R., 2007. Non-coding transcripts in the H19 imprinting control region mediate gene silencing in transgenic Drosophila. EMBO Rep. 8: 1068-1073.

Schwenk, F., Baron, U., and Rajewsky, K., 1995. A cre-transgenic mouse strain for the ubiquitous deletion of loxP-flanked gene segments including deletion in germ cells. Nucleic Acids Res. 23: 5080-5081.

Shi, Y., Lan, F., Matson, C., Mulligan, P., Whetstine, J.R., Cole, P.A., Casero, R.A., and Shi, Y., 2004. Histone demethylation mediated by the nuclear amine oxidase homolog LSD1. Cell 119: 941-953.

Shibata, H., Yoda, Y., Kato, R., Ueda, T., Kamiya, M., Hiraiwa, N., Yoshiki, A., Plass, C., Pearsall, R.S., Held, W.A., et al. 1998. A methylation imprint mark in the mouse imprinted gene Grf1/Cdc25Mm locus shares a common feature with the U2afbp-rs gene: An association with a short tandem repeat and a hypermethylated region. Genomics 49: 30-37.

Shin, J.Y., Fitzpatrick, G.V., and Higgins, M.J., 2008. Two distinct mechanisms of silencing by the KvDMR1 imprinting control region. EMBO T. 27: 168-178.

Sleutels, F., and Barlow, D.P. 2001. Investigation of elements sufficient to imprint the mouse Air promoter. Mol. Cell. Biol. 21: 5008-5017.

Sleutels, F., Zwart, R., and Barlow, D.P., 2002. The non-coding Air RNA is required for silencing autosomal imprinted genes. Nature 415: 810-813.

Sleutels, F., Tjon, G., Ludwig, T., and Barlow, D.P., 2003. Imprinted silencing of Slc22a2 and Slc22a3 does not need transcriptional overlap between Igf2r and Air. EMBO J. 22: 3696-3704.

Song, F., Smith, J.F., Kimura, M.T., Morrow, A.D., Matsuyama, T., Nagase, H., and Held, W.A., 2005. Association of tissuespecific differentially methylated regions (TDMs) with differential gene expression. Proc. Natl. Acad. Sci. 102: 33363341.

Stoger, R., Kubicka, P., Liu, C.G., Kafri, T., Razin, A., Cedar, H., and Barlow, D.P., 1993. Maternal-specific methylation of the imprinted mouse Igf2r locus identifies the expressed locus as carrying the imprinting signal. Cell 73: 61-71.

Takada, S., Paulsen, M., Tevendale, M., Tsai, C.E., Kelsey, G., Cattanach, B.M., and Ferguson-Smith, A.C., 2002. Epigenetic analysis of the Dlk1-Gtl2 imprinted domain on mouse chromosome 12: Implications for imprinting control from comparison with Igf2-H19. Hum. Mol. Genet. 11: 77-86.

Tremblay, K.D., Duran, K.L., and Bartolomei, M.S., 1997. A 5' 2 kilobase-pair region of the imprinted mouse H19 gene exhibits exclusive paternal methylation throughout development. Mol. Cell. Biol. 17: 4322-4329.

Valleley, E.M., Cordery, S.F., and Bonthron, D.T., 2007. Tissuespecific imprinting of the ZAC/PLAGL1 tumour suppressor gene results from variable utilization of monoallelic and biallelic promoters. Hum. Mol. Genet. 16: 972-981.

Williamson, C.M., Ball, S.T., Nottingham, W.T., Skinner, J.A., Plagge, A., Turner, M.D., Powles, N., Hough, T., Papworth, D., Fraser, W.D., et al. 2004. A cis-acting control region is required exclusively for the tissue-specific imprinting of Gnas. Nat. Genet. 36: 894-899.

Williamson, C.M., Turner, M.D., Ball, S.T., Nottingham, W.T., Glenister, P., Fray, M., Tymowska-Lalanne, Z., Plagge, A., Powles-Glover, N., Kelsey, G., et al. 2006. Identification of an 
imprinting control region affecting the expression of all transcripts in the Gnas cluster. Nat. Genet. 38: 350-355.

Wood, A.J., Roberts, R.G., Monk, D., Moore, G.E., Schulz, R., and Oakey, R.J., 2007. A screen for retrotransposed imprinted genes reveals an association between X chromosome homology and maternal germ-line methylation. PLoS Genet. 3: e20. doi: 10.1371/journal.pgen.0030020.

Yamada, Y., Watanabe, H., Miura, F., Soejima, H., Uchiyama, M., Iwasaka, T., Mukai, T., Sakaki, Y., and Ito, T., 2004. A comprehensive analysis of allelic methylation status of CpG islands on human chromosome 21q. Genome Res. 14: 247-266.

Yatsuki, H., Joh, K., Higashimoto, K., Soejima, H., Arai, Y., Wang, Y., Hatada, I., Obata, Y., Morisaki, H., Zhang, Z., et al. 2002. Domain regulation of imprinting cluster in Kip2/Lit1 subdomain on mouse chromosome 7F4/F5: Large-scale DNA methylation analysis reveals that DMR-Lit1 is a putative imprinting control region. Genome Res. 12: 1860-1870. 


\section{Erratum}

Genes \& Development 23: 105-117 (2008)

\section{Transcription is required for establishment of germline methylation marks at imprinted genes}

Mita Chotalia, Sebastien A. Smallwood, Nico Ruf, Claire Dawson, Diana Lucifero, Marga Frontera, Katherine James, Wendy Dean, and Gavin Kelsey

In the above-mentioned paper, there was an error in the primers used to detect the Kcnq1 transcript in mouse growing oocytes in Figure 5A. The Kcnq1 primers labelled Ex11-F and Ex12-R are in fact located in exons 10 and 11, respectively, so the RTPCR product for Kcnq1 does not span the DMR located in intron 11. The authors have repeated the RT-PCR analysis using new primers located in exons 10 and 12 (sequences given in updated Supplemental Table 1) and with this primer pair they detect Kcnq1 transcripts traversing the intron 11 DMR in mouse growing oocytes (as shown in the corrected Fig. 5, below), using the same conditions as in the previous assay. This correction does not alter the conclusion of the study in any way.

The authors apologize for the error.

A

Figure 5. Transcription across maternal germline DMRs in oocytes is common among imprinted genes. $(A$, left) Schemes representing the imprinted loci analyzedIgf2r, Grb10, Kcnq1, Zac1, and Impactshowing the locations of the primers used for RT-PCRs in relation to the germline DMRs (filled circles). The schemes are not to scale, and for simplicity, not all exons of the genes are shown. Characterized start sites or start sites determined by 5'RACE analysis are indicated by arrows, with those above the line representing start sites detected in somatic cells and those below the line representing start sites detected in oocytes. Novel exons identified by 5'RACE are shown as gray boxes. (Right) RT-PCRs for these loci for transcripts traversing the DMRs in day 5 (d5), day 10 (d10), day 15 (d15), and MII oocytes are shown (as in Fig. 1C). For both Igf2r and Grb10, RT-PCRs labelled with Ex1-F/ Ex3-R1 assay transcripts from the canonical somatic promoter and RT-PCRs labelled with Un1-F/Ex3-R2 assay the novel start sites identified in oocytes by $5^{\prime}$ RACE. The multiple bands for Zac1 reflect alternative splicing of the various $5^{\prime}$ untranslated exons, with exon 8 being the first coding exon. (B) RT-PCR analysis of transcripts for the H19 paternal germline DMR. The RT-PCR represents amplicon 4 from Schoenfelder et al. (2007). (C) RTPCR analysis of transcripts crossing the intragenic CGIs of four nonimprinted genes. Gene Sp6 represents locus PvuII 44, Chst8 is PstI 53, Sema6c is PstI 58, and Adra1b is PvulI 66 from Song et al. (2005). In the schemes to the left, the locations of the CGIs are represented by the open circles. In each panel, the lanes

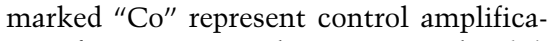
tion from E13.5 embryo RNA. The left lanes on each gel show a 100-bp marker ladder. (nd) Not done.

B

C
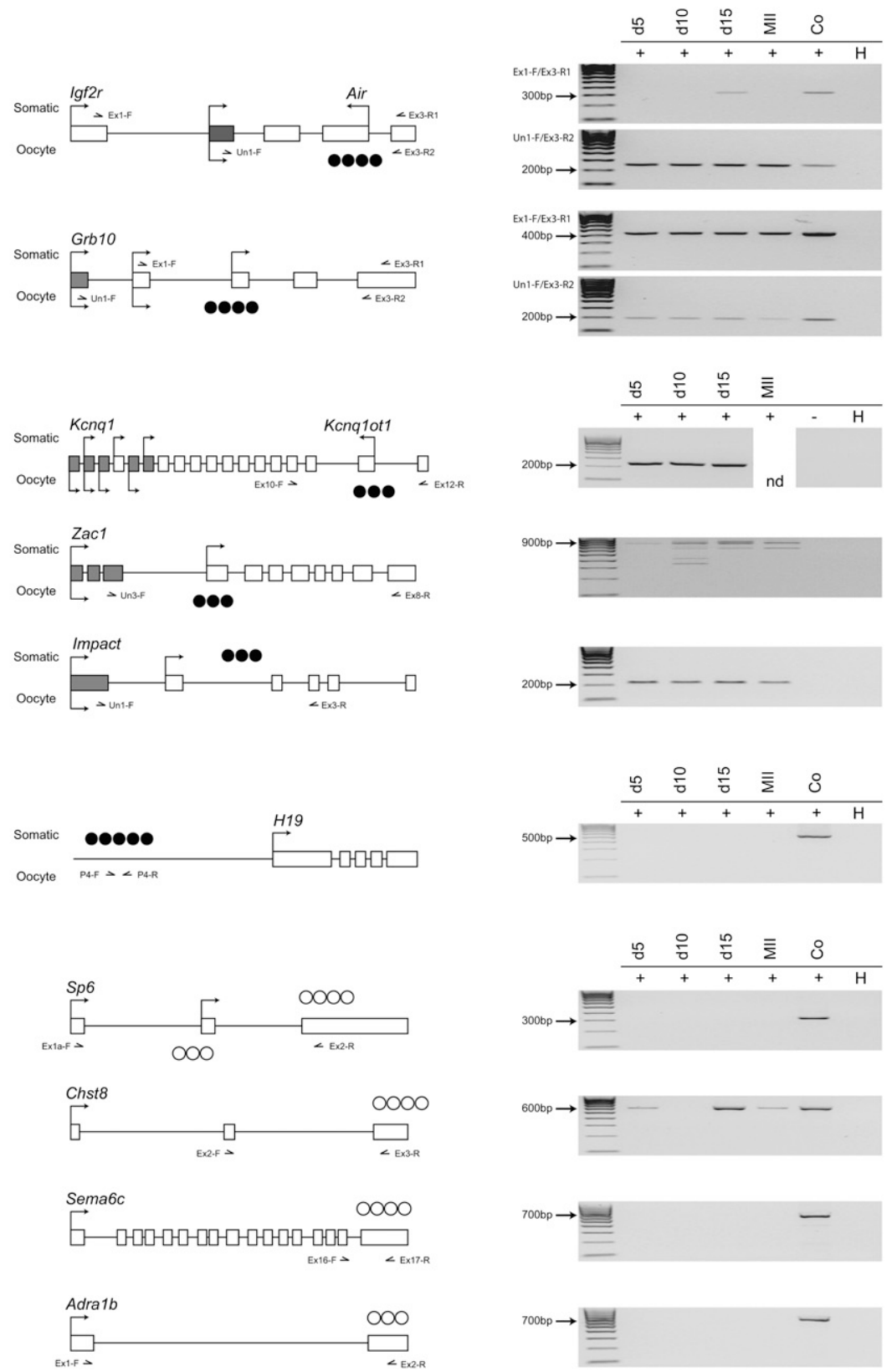
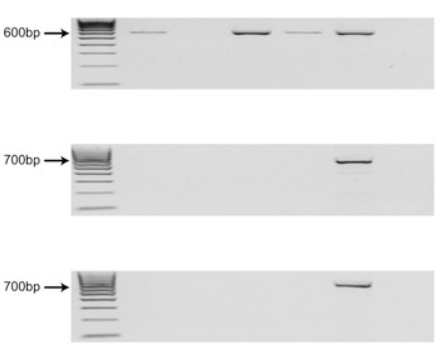


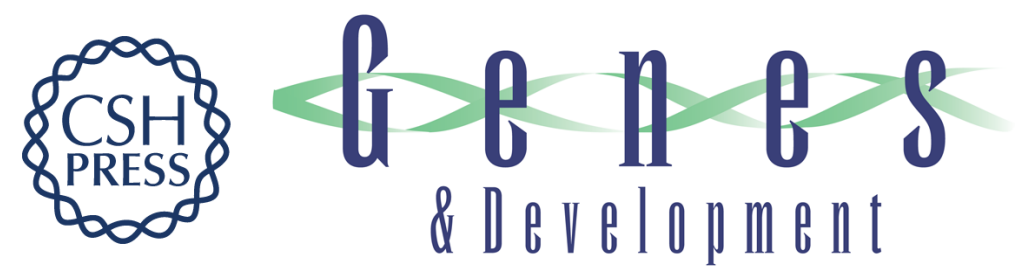

\section{Transcription is required for establishment of germline methylation marks at imprinted genes}

Mita Chotalia, Sebastien A. Smallwood, Nico Ruf, et al.

Genes Dev. 2009, 23:

Access the most recent version at doi:10.1101/gad.495809

\section{Supplemental http://genesdev.cshlp.org/content/suppl/2009/01/07/23.1.105.DC1 \\ Material}

Related Content Erratum: Transcription is required for establishment of germline methylation marks at imprinted genes

Mita Chotalia, Sebastien A. Smallwood, Nico Ruf, et al.

Genes Dev. October , 2009 23: 2358

References This article cites 58 articles, 14 of which can be accessed free at: http://genesdev.cshlp.org/content/23/1/105.full.html\#ref-list-1

Articles cited in:

http://genesdev.cshlp.org/content/23/1/105.full.html\#related-urls

\section{License}

Email Alerting

Service

Receive free email alerts when new articles cite this article - sign up in the box at the top right corner of the article or click here.

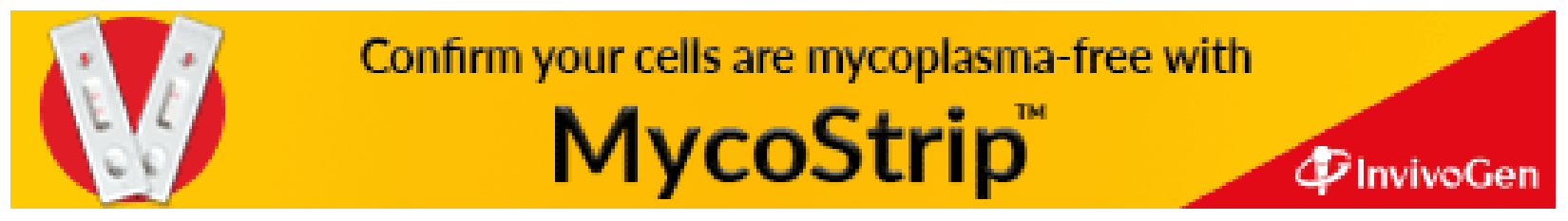

